\author{
NONCOMMUTATIVE HARMONIC ANALYSIS \\ WITH APPLICATIONS TO PROBABILITY II \\ BANACH CENTER PUBLICATIONS, VOLUME 89 \\ INSTITUTE OF MATHEMATICS \\ POLISH ACADEMY OF SCIENCES \\ WARSZAWA 2010
}

\title{
THE POWERS SUM \\ OF SPATIAL CPD-SEMIGROUPS AND CP-SEMIGROUPS
}

\author{
MICHAEL SKEIDE \\ Dipartimento S.E.G.e S., Università degli Studi del Molise \\ Via de Sanctis, 86100 Campobasso, Italy \\ E-mail: skeide@unimol.it \\ Homepage: http://www.math.tu-cottbus.de/INSTITUT/lswas/_skeide.html
}

\begin{abstract}
We define spatial CPD-semigroups and construct their Powers sum. We construct the Powers sum for general spatial CP-semigroups. In both cases, we show that the product system of that Powers sum is the product of the spatial product systems of its factors. We show that on the domain of intersection, pointwise bounded CPD-semigroups on the one side and Schur CP-semigroups on the other, the constructions coincide. This summarizes all known results about Powers sums and generalizes them considerably.
\end{abstract}

1. Introduction. At the 2002 AMS-Workshop on 'Advances in Quantum Dynamics' in Mount Holyoke, Powers described a sum operation for spatial $E_{0}$-semigroups on $\mathcal{B}(H)$, the algebra of bounded operators on a Hilbert space $H$. The result is a Markov semigroup and Powers asked for the product system of that Markov semigroup in the sense of Bhat [Bha96, and if that product system coincides or not with the tensor product of the Arveson systems of the $E_{0}$-semigroups. (By Arveson system we shall refer to product systems of Hilbert spaces as introduced by Arveson Arv89, while product system refers to the more general situation of Hilbert modules.)

Still during the workshop (see Skeide Ske03a]) we could show that the Arveson system of the Powers sum is our product of spatial product systems introduced in Ske06] (preprint 2001) immediately for Hilbert modules. In the case of Hilbert spaces, the product is a subsystem of the tensor product. (For modules there is no tensor product of product systems.) Liebscher [Lie03] showed that the product may but need not be all of the tensor product. The question if the subsystem of the tensor product is nevertheless isomorphic to the full tensor product or not, remained open until Powers [Pow04]: It need not.

2000 Mathematics Subject Classification: 46L55, 46L53, 60J25, 46L08.

Key words and phrases: quantum probability, quantum dynamics, product systems, spatial dynamics.

The paper is in final form and no version of it will be published elsewhere. 
The Powers sum has been generalized in several directions. Powers Pow04 generalized it to CP-semigroups that are spatial in his sense (a sense we consider too narrow). In Bhat, Liebscher and Skeide BLS08a we constructed the Powers sum for spatial $E_{0}$-semigroups on $\mathcal{B}^{a}(E)$, the algebra of adjointable operators on a Hilbert module $E$. We also showed that the product system of the sum is our product.

In Section 2 we introduce spatial CPD-semigroups and construct their spatial product systems. (This adds several new facts to CPD-semigroups and their GNS-systems as discussed in BBLS04. In particular, like discussed in [BLS08b for spatial CP-semigroups, the spatial product system of a CPD-semigroup, in the $C^{*}$-case, may be bigger than the GNS-system.) In Section 3 we construct a Powers sum for them, which is a spatial CPD-semigroup, too. We show that the product system of the sum is our product of the spatial product systems of the constituents. In Section 5 we introduce a Powers sum for spatial strict CP-semigroups acting on (not necessarily equal) $\mathcal{B}^{a}(E) \mathrm{s}$, and show also here that the spatial product systems of the sum is the product of the spatial product systems of the constituents. Both sorts of Powers sums include Powers construction [Pow04] (adding to Pow04 the identification of the Arveson system of the sum) and generalize it considerably. Our second Powers sum for CP-semigroups on $\mathcal{B}^{a}(E)$ includes and generalizes [BLS08a] and furnishes the case treated there, $E_{0}$-semigroups, with a more transparent proof. Finally, in Section 6 we show that the subclass of pointwise bounded CPD-semigroups and the subclass of Schur CP-semigroups are two sides of the same coin.

The discussion is mainly for $C^{*}$-algebras and modules. For the reasons explained in Section 2 , this case is more peculiar. With few modifications, also explained in Section 2 , the case of von Neumann algebras and modules is always included, usually, with simplified proofs.

It would be interesting to follow the story in the historical order. But for this we would have to introduce a lot of terminology, needed just to describe the known results, before we came to new ones. We prefer, therefore, to start immediately with the discussion of spatial CPD-semigroups and their product systems, followed by the definition of their Powers sum and the identification of its product system. Only then do we explain how this specializes to Powers results.

2. Spatial CPD-semigroups and their product systems. Let $S$ denote a set. We shall consider kernels $\mathfrak{K}: S \times S \rightarrow \mathcal{B}(\mathcal{A}, \mathcal{B}),\left(s, s^{\prime}\right) \mapsto \mathfrak{K}^{s, s^{\prime}}$ with values in the bounded maps between two $C^{*}$-algebras $\mathcal{A}$ and $\mathcal{B}$. In the case of von Neumann algebras we shall require the maps $\mathfrak{K}^{s, s^{\prime}}$ to be $\sigma$-weak. Following the definition in Barreto, Bhat, Liebscher and Skeide [BBLS04, we say a kernel is completely positive definite $(\boldsymbol{C P D})$ if

$$
\sum_{i, j} b_{i}^{*} \mathfrak{K}^{s_{i}, s_{j}}\left(a_{i}^{*} a_{j}\right) b_{j} \geq 0
$$

for all choices of finitely many elements $s_{i} \in S, a_{i} \in \mathcal{A}, b_{i} \in \mathcal{B}$. A typical example of a CPD-kernel is given by

$$
\mathfrak{K}^{s, s^{\prime}}:=\left\langle\xi^{s}, \bullet \xi^{s^{\prime}}\right\rangle
$$

for a family $\left(\xi^{s}\right)_{s \in S}$ of elements in a correspondence $E$ from $\mathcal{A}$ to $\mathcal{B}$. In fact, if $\mathcal{A}$ and $\mathcal{B}$ are unital, then every CPD-kernel can be recovered that way by its Kolmogorov 
decomposition. If we require that $E$ is generated by $\left(\xi^{s}\right)_{s \in S}$ as a correspondence, then the pair $\left(E,\left(\xi^{s}\right)_{s \in S}\right)$ is unique up to bilinear unitary equivalence. We refer to it as the Kolmogorov decomposition and to $E$ as the Kolmogorov correspondence of $\mathfrak{K}$.

2.1. REMARK. If $S$ has exactly one element, then the CPD-kernels on $S$ are precisely the CP-maps, and the Kolmogorov decomposition is Paschke's GNS-construction for CPmaps; see Pas73]. If $S=\{1, \ldots, n\}$, then the CPD-kernels on $S$ can be identified with Schur CP-maps from $M_{n}(\mathcal{A})$ to $M_{n}(\mathcal{B})$ that act matrix elementwise as $a_{i j} \mapsto \mathfrak{K}^{i, j}\left(a_{i, j}\right)$. If $\mathcal{A}=\mathbb{C}$, then by $\left(s, s^{\prime}\right) \mapsto \mathfrak{K}^{s, s^{\prime}}(1)$ we establish a one-to-one correspondence with positive definite $\mathcal{B}$-valued kernels. If also $\mathcal{B}=\mathbb{C}$, then we recover the usual Kolmogorov decomposition for $\mathbb{C}$-valued kernels.

2.2. Remark. Even if $\mathcal{A}$ and $\mathcal{B}$ are nonunital, we get a correspondence $E$ from $\mathcal{A}$ to $\mathcal{B}$ and elements $\xi^{a, s}$ such that $\mathfrak{K}^{s, s^{\prime}}\left(a^{*} a^{\prime}\right)=\left\langle\xi^{a, s}, \xi^{a^{\prime}, s^{\prime}}\right\rangle$. But it is, in general, impossible to obtain suitable elements $\xi^{s}$. It is possible to unitalize the kernel to the unitalizations $\widetilde{\mathcal{A}}$ and $\widetilde{\mathcal{B}}$ by the unitalization procedure in Skeide [Ske08] or, if all $\mathfrak{K}^{s, s^{\prime}}$ are strict, to the multiplier algebras.

A $\boldsymbol{C P D}$-semigroup is a family $\mathfrak{T}=\left(\mathfrak{T}_{t}\right)_{t \in \mathbb{R}_{+}}$of $\mathcal{B}(\mathcal{B})$-valued CPD-kernels on $S$ such that for all $s, s^{\prime} \in S$ the maps $\mathfrak{T}_{t}^{s, s^{\prime}}$ form a semigroup on $\mathcal{B}$. If all these semigroups are continuous in a certain topology, then we say the CPD-semigroup is continuous in that topology.

The same way CPD-kernels are related to correspondences via Kolmogorov decomposition, CPD-semigroups are related to product systems of correspondences via a GNStype construction. Following Bhat and Skeide [BS00, a product system is a family $E^{\odot}=\left(E_{t}\right)_{t \in \mathbb{R}_{+}}$of correspondences over $\mathcal{B}$ such that $E_{0}=\mathcal{B}$, with a family of bilinear unitaries $u_{s, t}: E_{s} \odot E_{t} \rightarrow E_{s+t}$ such that the product $x_{s} y_{t}:=u_{s, t}\left(x_{s} \odot y_{t}\right)$ is associative, and such that $u_{0, t}$ and $u_{t, 0}$ are the canonical identifications. If $\mathcal{B}$ is unital, a unit for $E^{\odot}$ is a family $\xi^{\odot}=\left(\xi_{t}\right)_{t \in \mathbb{R}_{+}}$of elements $\xi_{t} \in E_{t}$ such that $\xi_{0}=\mathbf{1}$ and $\xi_{s} \xi_{t}=\xi_{s+t}$. If $\left(\xi^{\odot} \odot\right)_{s \in S}$ is a family of units for $E^{\odot}$, then by the definition of the internal tensor product $E_{s} \odot E_{t}$ it follows that the maps

$$
\mathfrak{T}_{t}^{s, s^{\prime}}:=\left\langle\xi_{t}^{s}, \bullet \xi_{t}^{s^{\prime}}\right\rangle
$$

form a semigroup. Clearly, the family of kernels $\left(s, s^{\prime}\right) \mapsto \mathfrak{T}_{t}^{s, s^{\prime}}$ forms a CPD-semigroup. By BBLS04, every CPD-semigroup for unital $\mathcal{B}$ arises in that way. If $E^{\odot}$ is generated as a product system by the family of units $\left(\xi^{s}\right)_{s \in S}$, then the pair $\left(E^{\odot},\left(\xi^{s}\right)_{s \in S}\right)$ is

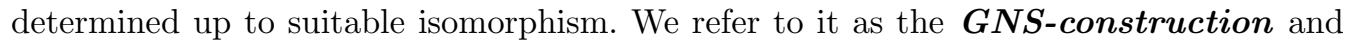
to $E^{\odot}$ as the $\boldsymbol{G N S}$-system of $\mathfrak{T}$.

2.3. REMARK. If $S$ has one element, then the CPD-semigroups on $S$ are precisely the CP-semigroups and the GNS-construction is that from BS00.

2.4. Observation. If $E^{\odot}$ is the GNS-system of a CPD-semigroup $\mathfrak{T}$ and if $\left(\xi^{\odot}\right)_{s \in S}$ is the generating family of units, then

$$
E_{t}=\overline{\operatorname{span}}\left\{b_{n} \xi_{t_{n}}^{s_{n}} \odot \ldots \odot b_{1} \xi_{t_{1}}^{s_{1}} b_{0} \mid n \in \mathbb{N} ; s_{1}, \ldots, s_{n} \in S ; b_{0}, \ldots, b_{n} \in \mathcal{B} ; t_{1}+\ldots+t_{n}=t\right\} .
$$


This will help us to identify, later on, the spatial product system of the Powers sum of spatial CPD-semigroups on $\mathcal{B}$ or spatial CP-semigroups on $\mathcal{B}^{a}(E)$.

The definitions and results repeated so far were for (unital) $C^{*}$-algebras and $C^{*}$-modules or correspondences. They adapt easily to von Neumann algebras, modules, and correspondences, if: 1) We require maps on or between von Neumann algebras to be $\sigma$-weak. 2) We replace the tensor product by its strongly closed version. Without further mention, we assume these conventions when we speak about the von Neumann case.

The following definition generalizes Arveson's Arv97 for (normal) CP-semigroups on $\mathcal{B}(G)$ ( $G$ some Hilbert space). It is new, except for the part on domination of CPDsemigroups from BBLS04. The version for CP-semigroups on $C^{*}$-algebras is from Bhat, Liebscher and Skeide [BLS08b; that for von Neumann algebras $\mathcal{B} \subset \mathcal{B}(G)$ from Skeide Ske09a.

2.5. Definition. Let $\mathcal{B}$ be a unital $C^{*}$-algebra (a von Neumann algebra) and let $S$ be a set.

A CPD-semigroup $\mathfrak{T}$ on $S$ with values in $\mathcal{B}(\mathcal{B})$ dominates another $\mathfrak{S}$ if the kernels $\mathfrak{T}_{t}-\mathfrak{S}_{t}$ are CPD for all $t \in \mathbb{R}_{+}$. In this situation we write $\mathfrak{T} \geq \mathfrak{S}$.

A CPD-semigroup $\mathfrak{S}$ is elementary if it has the form $\mathfrak{S}_{t}^{s, s^{\prime}}=c_{t}^{s *} \bullet c_{t}^{s^{\prime}}$ for a family $\left(c^{s}\right)_{s \in S}$ of (strongly) continuous semigroups $c^{s}=\left(c_{t}^{s}\right)_{t \in \mathbb{R}_{+}}$in $\mathcal{B}$.

A unit for a CPD-semigroup $\mathfrak{T}$ is an elementary CPD-semigroup $\mathfrak{S}$ such that $\mathfrak{T} \geq \mathfrak{S}$.

A CPD-semigroup $\mathfrak{T}$ is spatial if it admits a unit. If we wish to emphasize the choice of the unit, we will also speak of the pair $(\mathfrak{T}, \mathfrak{S})$ as a spatial CPD-semigroup.

2.6. Remark. As pointed out in BLS08b, semigroups of elements $c_{t}$ in a unital $C^{*}$-algebra that are continuous in any of the natural topologies are uniformly continuous automatically. Indeed, if $c_{t}$ is weakly continuous, then the semigroup $b \mapsto b c_{t}$ of maps in $\mathcal{B}(\mathcal{B})$ it is weakly continuous and, therefore, strongly continuous. In particular, the family $c_{t}=\mathbf{1} c_{t} \in \mathcal{B}$ is norm continuous. (If $\mathcal{B}$ is nonunital, then it makes no sense to speak of a semigroup in $\mathcal{B}$ indexed by $t \geq 0$, but only $t>0$.) The strong topology of a von Neumann algebra $\mathcal{B} \subset \mathcal{B}(G)$ is much weaker and allows for semigroups with unbounded generator.

Note, too, that spatiality without continuity conditions on the unit $\mathfrak{S}$ is a trivial issue. In fact, the zero-semigroup $\mathfrak{O}$ defined by $\mathfrak{O}_{t}^{s, s^{\prime}}=0$ for all $s, s \in S$ and all $t>0$ would be a unit for every CPD-semigroup on $S$.

In the sequel, 'strongly continuous' for a semigroup $T$ of maps on a $C^{*}$-algebra $\mathcal{B}$ that is not represented as an algebra of operators on a Hilbert space or, more generally, on a Hilbert module, means that $t \mapsto T_{t}(b)$ is norm continuous for every $b \in \mathcal{B}$. If $T$ acts on a von Neumann algebra $\mathcal{B} \subset \mathcal{B}(G)$, then it means that $t \mapsto T_{t}(b) g$ is norm continuous for all $b \in \mathcal{B}, g \in G$. The same convention applies to semigroups acting on $\mathcal{B}^{a}(E)$. We see in a minute that spatial strongly continuous $\mathrm{CPD}$-semigroups on an abstract $C^{*}$-algebra are even uniformly continuous. So, in these notes, where we are interested only in spatial CPD-semigroups, we will, usually, use 'strongly continuous' only when we speak about operator algebras $\mathcal{B} \subset \mathcal{B}(G)$ or $\mathcal{B}^{a}(E)$. 
2.7. Definition ( Ske06]). A spatial product system is a pair $\left(E^{\odot}, \omega^{\odot}\right)$ consisting of a product system and a central unital reference unit $\omega^{\odot}$ (that is, all $\omega_{t}$ commute with all $b \in \mathcal{B}$ and all $\omega_{t}$ are unit vectors in the sense that $\left\langle\omega_{t}, \omega_{t}\right\rangle=\mathbf{1}$ ).

In general, we will call a product system spatial if it has central unital units. Note, however, that the spatial structure may depend on the choice of the reference unit. Spatial product systems and their product (see Section 3) have been introduced in Skeide Ske06. They form a subcategory of product systems which behaves best in analogy with Arveson's classification scheme for Arveson systems. (There is an index for spatial product systems and the index behaves additively under the product of spatial product systems; see [Ske06]. A tensor product of product systems does, in general, not exist.)

2.8. TheOrem. For a strongly continuous $C P D$-semigroup $\mathfrak{T}$ on $S$ with values in $\mathcal{B}(\mathcal{B})$ for a unital $C^{*}$-algebra $\mathcal{B}$ the following conditions are equivalent:

1. $\mathfrak{T}$ is spatial.

2. The (continuous) GNS-system of $\mathfrak{T}$ embeds into a (continuous) spatial product system. In particular, $\mathfrak{T}$ is uniformly continuous.

3. $\mathfrak{T}$ has a Christensen-Evans generator, that is, $\mathfrak{L}^{s, s^{\prime}}:=\left.\frac{d \mathfrak{T}^{s, s^{\prime}}}{d t}\right|_{t=0}$ exists for all $s, s^{\prime} \in S$ and there are a CPD-kernel $\mathfrak{L}_{0}$ and elements $\beta_{s} \in \mathcal{B}$ such that

$$
\mathfrak{L}^{s, s^{\prime}}(b)=\mathfrak{L}_{0}^{s, s^{\prime}}(b)+b \beta_{s^{\prime}}+\beta_{s}^{*} b .
$$

Proof. The proof is very much like the proofs of BLS08b, Theorem 3.4 and Corollary 3.7], just with more indices. (The number of indices is $\# S+1$.) In so far, we need to explain only the construction of the extended CPD-semigroup on $S_{0}:=S \cup\{0\}$, and we say a word on what continuous product systems means.

We start with the latter. If a CPD-semigroup is strongly continuous, then its product system is continuous in the sense of Skeide Ske03b. By Ske03b, Theorem 7.7], if a continuous product system has a single unit $\xi^{\odot}$ such that the CP-semigroup $\left\langle\xi_{t}, \bullet \xi_{t}\right\rangle$ is uniformly continuous, then all semigroups $\left\langle\xi_{t}, \bullet \xi_{t}^{\prime}\right\rangle$ are uniformly continuous. And the reference unit $\omega^{\odot}$ generates the trivial semigroup which is uniformly continuous.

The basic observation for constructing the spatial product system into which the GNS-system embeds, is the following. Let $\mathfrak{S}$ be an elementary CPD-semigroup on $S$ generated by semigroups $c^{s}$ in $\mathcal{B}$, and suppose that $\mathfrak{T}$ dominates $\mathfrak{S}$. Then the semigroup $\widehat{\mathfrak{T}}$ on $S_{0}$ defined by setting

$$
\widehat{\mathfrak{T}}_{t}^{s, s^{\prime}}:=\mathfrak{T}_{t}^{s, s^{\prime}}, \quad \quad \widehat{\mathfrak{T}}_{t}^{0, s}:=\bullet c_{t}, \quad \widehat{\mathfrak{T}}_{t}^{s, 0}:=c_{t}^{*} \bullet,
$$

is CPD. (It can be written as the sum of the extension of $\mathfrak{T}_{t}-\mathfrak{S}_{t}$ from $S$ to $S_{0}$ by 0 , and a suitable elementary CPD-semigroup on $S_{0}$; see [BLS08b.) Clearly, the GNS-system of $\widehat{\mathfrak{T}}$ is spatial (the unit $\xi^{0}$ is central and unital), and it contains the GNS-system of $\mathfrak{T}$; see [BLS08b] for details.

2.9. REMARK. In general, the generators of uniformly continuous CPD-semigroups with values in $\mathcal{B}(\mathcal{B})$ are precisely the conditionally completely positive definite (CCPD) $\mathcal{B}(\mathcal{B})$-valued kernels (that is, the kernel fulfills 2.1 under the condition that $\sum_{i} a_{i} b_{i}=0$ ); see BBLS04. Like for CP-semigroups on a $C^{*}$-algebra, boundedness of the generator is not sufficient for having Christensen-Evans form. 
It is an open problem whether or not the spatial extension of the GNS-system of $\mathfrak{T}$ constructed in the proof of Theorem 2.8 depends on the choice of the unit $\mathfrak{S}$. On the other hand, it is easy to see that it does not depend on the choice of the implementing semigroup c. (In fact, two semigroups in $\mathcal{B}$ implementing the same elementary CP-semigroup on $\mathcal{B}$ can differ at most by a unitary semigroup in the center of $\mathcal{B}$. Using this, it is more or less obvious to see that the GNS-system of the extended kernel $\widehat{\mathfrak{T}}$ does not change under such a variation.) Henceforth, we call it the spatial extension of the GNS-system based on the unit $\mathfrak{S}$. Fortunately, the Powers sum of CPD-semigroups depends from the beginning on the choice of units. So, it is not tragic if also the spatial extensions of their GNS-systems would depend on that choice.

For von Neumann algebras the situation is much better:

2.10. TheOREM. For a strongly continuous $C P D$-semigroup $\mathfrak{T}$ on $S$ with values in $\mathcal{B}(\mathcal{B})$ for a von Neumann algebra $\mathcal{B} \subset \mathcal{B}(G)$ the following conditions are equivalent:

1. $\mathfrak{T}$ is spatial.

2. The GNS-system of $\mathfrak{T}$ is spatial.

Proof. The proof is the same as in Skeide Ske09a] for CP-semigroups. It cannot be reproduced here for reasons of space. (Very roughly, the idea is that for von Neumann algebras there is an order isomorphism from the partially ordered set of positive contraction endomorphisms of the GNS-system of a CPD-semigroup $\mathfrak{T}$ to the partially ordered set of CPD-semigroups dominated by $\mathfrak{T}$; see BBLS04. And the range of the positive contraction morphism corresponding to a unit $\mathfrak{S}$ is just the one-dimensional product system $(\mathcal{B})_{t \in \mathbb{R}_{+}}$, which contains the central unital unit $\left.(\mathbf{1})_{t \in \mathbb{R}_{+}} \cdot\right)$

3. The Powers sum of spatial CPD-semigroups. Let $\mathfrak{T}^{1}$ and $\mathfrak{T}^{2}$ be spatial CPD-semigroups on sets $S^{1}$ and $S^{2}$, respectively, with values in $\mathcal{B}(\mathcal{B})$. Choose units $\mathfrak{S}^{1}$ and $\mathfrak{S}^{2}$ for them implemented by semigroups $c^{1}$ and $c^{2}$, respectively, in $\mathcal{B}$. Define a semigroup $\mathfrak{T}:=\mathfrak{T}^{1} \boxplus \mathfrak{T}^{2}$ on $S:=S^{1} \uplus S^{2}$ (disjoint union), by setting

$$
\begin{aligned}
\left(\mathfrak{T}^{1} \boxplus \mathfrak{T}^{2}\right)^{s, s^{\prime}}:=\left(\mathfrak{T}^{i}\right)^{s, s^{\prime}} & \left(i=1,2 ; s, s^{\prime} \in S^{i}\right), \\
\left(\mathfrak{T}^{1} \boxplus \mathfrak{T}^{2}\right)^{s_{i}, s_{j}}:=\left(c^{i}\right)^{s_{i}{ }^{*}} \bullet\left(c^{j}\right)^{s_{j}} & \left(i \neq j, s_{i} \in S^{i}, s_{j} \in S^{j}\right) .
\end{aligned}
$$

Observe that each $\mathfrak{S}^{i}$ is itself a spatial CPD-semigroup with unit $\mathfrak{S}^{i}$. Therefore, the definition applies also to $\mathfrak{S}:=\mathfrak{S}^{1} \boxplus \mathfrak{S}^{2}$.

3.1. THEOREM. $\mathfrak{T}$ is a spatial CPD-semigroup with unit $\mathfrak{S}$. Clearly, $\mathfrak{T}$ is (strongly) continuous if and only if each $\mathfrak{T}^{i}$ is (strongly) continuous.

Proof. We shall show $\mathfrak{T} \geq \mathfrak{S}$. This settles both that $\mathfrak{T}_{t}$ is CPD (as sum of the CPD-kernels $\mathfrak{T}_{t}-\mathfrak{S}_{t}$ and $\left.\mathfrak{S}_{t}\right)$ and that $\mathfrak{S}$ is a unit for $\mathfrak{T}$. We find

$$
\begin{aligned}
\left(\mathfrak{T}_{t}-\mathfrak{S}_{t}\right)^{s, s^{\prime}} & =\left(\mathfrak{T}_{t}^{i}-\mathfrak{S}_{t}^{i}\right)^{s, s^{\prime}} & & \left(i=1,2 ; s, s^{\prime} \in S^{i}\right), \\
\left(\mathfrak{T}_{t}-\mathfrak{S}_{t}\right)^{s_{i}, s_{j}} & =0 & & \left(i \neq j, s_{i} \in S^{i}, s_{j} \in S^{j}\right) .
\end{aligned}
$$

Since each $\mathfrak{T}_{t}^{i}-\mathfrak{S}_{t}^{i}$ is $\mathrm{CPD}$ on $S^{i}$ and since all mixing terms $s^{i} \in S^{i}, s^{j} \in S^{j}(i \neq j)$ disappear, this shows that $\mathfrak{T}_{t}-\mathfrak{S}_{t}$ is $\mathrm{CPD}$. 
3.2. Definition. We refer to $(\mathfrak{T}, \mathfrak{S}):=\left(\mathfrak{T}^{1}, \mathfrak{S}^{1}\right) \boxplus\left(\mathfrak{T}^{2}, \mathfrak{S}^{2}\right)$ as the Powers sum of $\left(\mathfrak{T}^{1}, \mathfrak{S}^{1}\right)$ and $\left(\mathfrak{T}^{2}, \mathfrak{S}^{2}\right)$.

We now wish to identify the spatial extension of the GNS-system of $(\mathfrak{T}, \mathfrak{S})$ as the product of the spatial extensions of the GNS-systems of $\left(\mathfrak{T}^{i}, \mathfrak{S}^{i}\right)$. To that goal we repeat the characterization in Skeide Ske06 of the product in terms of a universal property.

3.3. Theorem and Definition ( $\underline{\text { Ske06}}$, Theorem 5.1 and Definition 5.2]). Let $\left(E^{1^{\odot}}, \omega^{1}{ }^{\odot}\right.$ ) and $\left(E^{2 \odot}, \omega^{2}{ }^{\odot}\right)$ denote spatial product systems. Then there exists a spatial product system $\left(F^{\odot}, \omega^{\odot}\right)$ fulfilling the following properties:

1. $\left(F^{\odot}, \omega^{\odot}\right)$ contains $\left(E^{i}{ }^{\odot}, \omega^{i \odot}\right)(i=1,2)$ as spatial subsystems and is generated by them, that is, $F_{t}$ is spanned by expressions like

$$
x_{t_{n}}^{n} \odot \ldots \odot x_{t_{1}}^{1},
$$

$n \in \mathbb{N}, t_{1}+\ldots+t_{n}=t$, and $x_{t_{j}}^{j} \in E_{t_{j}}^{1} \cup E_{t_{j}}^{2}$.

2. The inner product of members $x^{1} \in E_{t}^{1} \subset F$ and $x^{2} \in E_{t}^{2} \subset F$ is given by

$$
\left\langle x^{1}, x^{2}\right\rangle=\left\langle x^{1}, \omega_{t}^{1}\right\rangle\left\langle\omega_{t}^{2}, x^{2}\right\rangle .
$$

Moreover, every spatial product system fulfilling these properties is canonically isomorphic to $\left(F^{\odot}, \omega^{\odot}\right)$. We call $\left(F^{\odot}, \omega^{\odot}\right)$ the product of the factors $\left(E^{i \odot}, \omega^{i \odot}\right)$ and we denote it by $\left(\left(E^{1} \odot E^{2}\right)^{\odot}, \omega^{\odot}\right)$.

Note that, by Property 2 , in the product the two reference units $\omega^{i \odot}$ of the factors get identified with the reference unit $\omega^{\odot}$.

3.4. TheOREm. The spatial extension of the GNS-system of $(\mathfrak{T}, \mathfrak{S}):=\left(\mathfrak{T}^{1}, \mathfrak{S}^{1}\right) \boxplus\left(\mathfrak{T}^{2}, \mathfrak{S}^{2}\right)$ is (spatially) isomorphic to the product of the spatial extensions of the GNS-systems of $\left(\mathfrak{T}^{1}, \mathfrak{S}^{1}\right)$ and $\left(\mathfrak{T}^{2}, \mathfrak{S}^{2}\right)$.

Proof. Recall that spatially isomorphic means that the isomorphism identifies also the reference units.

In Observation 2.4 we indicated a spanning subset of the GNS-system of a CPDsemigroup. We apply this to the GNS-system of the spatial extension of $(\mathfrak{T}, \mathfrak{S})$. Observe that the pieces of units $\xi_{t_{j}}^{j}$ come either from the GNS-system of $\mathfrak{T}^{1}$ or from the GNSsystem of $\mathfrak{T}^{2}$ or from the component 0 in $S \cup\{0\}$, that is from the reference unit of the GNS-system of the spatial extension of $(\mathfrak{T}, \mathfrak{S})$. One easily verifies that the inner product does not change if instead we replace that reference unit with one (no matter which) of the reference units of the spatial extensions of the GNS-systems of one of the factors. This shows that the spatial extension of the GNS-system of $(\mathfrak{T}, \mathfrak{S})$ contains the spatial extensions of the GNS-systems of the factors as subsets and is generated by them, as required in Property 1 of Theorem 3.3. It is also easy to check that the inner products of elements from different factors are those required by Property 2 of Theorem 3.3

3.5. Remark. Notation and formulation of the results is for the $C^{*}$-case. But this case is the more complicated one because the GNS-system of a spatial CPD-semigroup need not be spatial. With the standard topological conventions we applied in the preceding section, all statements (some of them in a simpler form) remain valid in the von Neumann case. 
3.6. Remark. It is clear that both product of spatial product systems and Powers sum of spatial CPD-semigroups may be carried out for families of products systems or of CPD-semigroups indexed by arbitrary sets. For spatial product systems this is mentioned in Ske06, Remark 5.7]. For spatial CPD-semigroups this means that for a family $\left(\mathfrak{T}^{\alpha}, \mathfrak{S}^{\alpha}\right)_{\alpha \in A}$ there is a Powers sum $\boxplus_{\alpha \in A} \mathfrak{T}^{\alpha}$. Of course, also the statement of the theorem remains valid for such families: The spatial extension of the GNS-system of the Powers sum is the product of the spatial extensions of the GNS-systems of the constituents.

4. Some special cases. In this section we discuss some examples. We have a look at how Schur semigroups of positive definite kernels are included. In Remark 4.2, we explain why such semigroups do not make sense in a noncommutative context, underlining CPD-semigroups as the correct generalization. In Example 4.3 we discuss how the case of finite sets can be described equivalently in terms of Schur CP-semigroups on matrix algebras. This includes all variants discussed by Powers for the case $\mathcal{B}=\mathcal{B}(G)$ and even generalizes them in that case. It does not cover the case studied in Bhat, Liebscher and Skeide [BLS08a], but it gives us a hint what to do in the following section.

4.1. Example (Semigroups of positive definite kernels). If $\mathcal{B}=\mathbb{C}$, we recover the notion of positive definite $(\boldsymbol{P D}) \mathbb{C}$-valued kernels and their Schur semigroups. In fact, a map $\mathfrak{K}^{s, s^{\prime}}$ on $\mathbb{C}$ is determined by the value $\mathfrak{k}^{s, s^{\prime}}:=\mathfrak{K}^{s, s^{\prime}}(1)$ and a $\mathbb{C}$-valued kernel $\mathfrak{k}$ on $S$ defines a $\mathcal{B}(\mathbb{C})$-valued kernel $\mathfrak{K}$ by setting $\mathfrak{K}^{s, s^{\prime}}(z):=\mathfrak{k}^{s, s^{\prime}} z$. Clearly, $\mathfrak{K}$ is CPD if and only if $\mathfrak{k}$ is PD.

The Schur product of two $\mathbb{C}$-valued kernels on $S$ is simply the pointwise product of functions on $S \times S$. Clearly, the Schur product is reflected by the composition of the corresponding $\mathcal{B}(\mathbb{C})$-valued kernels. Everything we know about CPD-semigroups has, thus, an immediate interpretation in terms of PD-semigroups: 1) The Schur product preserves PD. 2) PD-semigroups have a product system of Hilbert spaces (that is, an Arveson system) as GNS-system. 3) This Arveson system is generated by its units and, therefore, Fock. This fact has already been noted by Parthasarathy and Schmidt PS72]. They applied this knowledge to the PD-semigroup $\mathfrak{k}_{t}^{s, s^{\prime}}:=\int e^{i\left(s-s^{\prime}\right)} \mu_{t}(d t)$ on $\mathbb{R}$ that arises from the convolution semigroup $\left(\mu_{t}\right)_{t \in \mathbb{R}_{+}}$of distributions of a Lévy process, which enabled them to represent every Lévy process on the Fock space.

The product of Arveson systems of Fock type (so-called type I Arveson systems) is simply their tensor product. (This need not be so for non-type I spatial Arveson systems, so-called type II Arveson systems.) Tensor products of units in the factors give rise to units in the tensor product, and every unit in the tensor product arises that way. In order to understand the PD-semigroup on $S^{1} \uplus S^{2}$ it is better to assume that in each factor a reference unit has been distinguished, that corresponds to $0 \in S^{i} \cup\{0\}$. The set $S^{1} \uplus S^{2}$ is identified with the subset $\left(S^{1} \times\{0\}\right) \cup\left(\{0\} \times S^{2}\right)$ of the generating set $\left(S^{1} \cup\{0\}\right) \times\left(\{0\} \cup S^{2}\right)$ of units in the product. If both kernels come from Lévy processes, then the product simply describes the two processes as a pair of independent Lévy processes (or a two-dimensional Lévy process) on the same probability space (the product space). 
The structure of the units as products of the units of the factors remains valid for the product of arbitrary spatial product systems; see [Ske06, Theorem 5.6]. That the disjoint union of two sets $S^{1}$ and $S^{2}$ is, by the very definition of disjoint union, a subset of product of dotted sets $S^{1} \cup\{0\}$ and $\{0\} \cup S^{2}$, is reflected in a striking way by the structure of the set of units in the product.

4.2. REMARK. It is natural to ask for a notion of PD-semigroups of $\mathcal{B}$-valued kernels, sitting somehow in between $\mathbb{C}$-valued PD-kernels and general CPD-kernels. However, among PD-kernels there is no product operation whatsoever that would respect the PDcondition, as soon as the algebra $\mathcal{B}$ is noncommutative.

This is a central thread of positivity in a noncommutative setting: If we wish to compose positive noncommuting things in a positivity preserving way then composition must be composition of maps on the *-algebra. Almost never can it be based on multiplication of positive elements in a $*$-algebra.

The basic feature of positive things is that they possess some kind of square root. A positive element $a$ in a $C^{*}$-algebra can be written as $b^{*} b$. But, if there is another one $a^{\prime}=b^{*} b^{\prime}$, then $a a^{\prime}=b^{*} b b^{* *} b^{\prime}$ is almost never positive. An element that is positive, is $b^{*} b^{*} b^{\prime} b$. However, this element depends manifestly on the choice of the square roots $b$ and $b^{\prime}$.

A way out is to consider, from the beginning, the map $T=b^{*} \bullet b$ instead of $a=T(\mathbf{1})$. The knowledge of $T(\mathbf{1})$ is only rarely a suitable substitute for the whole map $T$. But, once we have that map, we may compose it with $T^{\prime}$, and, in fact, we get $T \circ T^{\prime}=\left(b^{\prime} b\right)^{*} \bullet\left(b^{\prime} b\right)$.

Going one step further to CP-type maps (for instance, CPD-kernels), one sees that the related GNS-constructions play the role of the square roots which may be multiplied. The multiplication is simply the tensor product of the associated GNS-correspondences; see [BS00, Observation 2.17] or [BBLS04, Observation 3.4.3]. To say it more provocatively: GNS-systems are square roots of the CPD-semigroups they stem from.

4.3. EXAmPle (Schur CP-semigroups on $M_{n}(\mathcal{B})$ ). Recall that the case of a CPD-semigroup on a one-point set $S$ is precisely the case of a CP-semigroup. More generally, a CPD-semigroup on an $n$-point set $S=\{1, \ldots, n\}$ gives rise to a CP-semigroup $T^{n}$ on $M_{n}(\mathcal{B})$, by setting

$$
\left(T_{t}^{n}(A)\right)_{i j}=\mathfrak{T}_{t}^{i, j}\left(a_{i j}\right) .
$$

Clearly, we do not obtain all CP-semigroups on $M_{n}(\mathcal{B})$ in that way. In fact, $T^{n}$ is a $\boldsymbol{S c h u r}$ semigroup, that is, it acts matrix elementwise on the matrix $A=\left(a_{i j}\right)$. So, what we really have is a one-to-one correspondence between CPD-semigroups on a fixed $n$-point set $S$ and Schur CP-semigroups on $M_{n}(\mathcal{B})$.

The elementary CP-semigroups on $M_{n}(\mathcal{B})$ which are also Schur semigroups are precisely those that are generated as $S_{t}^{n}=C_{t}^{*} \bullet C_{t}$ by semigroups $C=\left(C_{t}\right)_{t \in \mathbb{R}_{+}}$in $M_{n}(\mathcal{B})$ with diagonal matrices $C_{t} \in M_{n}(\mathcal{B})$. It is easy to check that a Schur CP-semigroup is spatial if and only if the corresponding CPD-semigroup $\mathfrak{T}$ on the $n$-point set $S$ is spatial. (The entries of the diagonal generate the elementary CPD-semigroup $\mathfrak{S}$ dominated by $\mathfrak{T}$, and vice versa.) 
We see that there is a Powers sum of spatial Schur CP-semigroups $T^{n_{i}}$ on $M_{n_{i}}(\mathcal{B})$ that provides a Schur CP-semigroup $T^{n_{1}} \boxplus T^{n_{2}}$ acting on $M_{n_{1}+n_{2}}(\mathcal{B})$.

The special case $n_{1}=n_{2}=1$ (semigroups on $\mathcal{B}$ having a semigroup on $M_{2}(\mathcal{B}$ ) as sum) includes all cases discussed by Powers with $\mathcal{B}=\mathcal{B}(G)$. (In that case, when $G$ is infinite-dimensional and separable, $M_{2}(\mathcal{B}(G))=\mathcal{B}(G \oplus G)$ and $G \oplus G \cong G$. This abuse, mixing a true binary operation among semigroups on $\mathcal{B}(G)$ with a binary operation among conjugacy classes, is quite common. For more general algebras where, usually, $M_{2}(\mathcal{B}) \nsucceq \mathcal{B}$, this is no longer possible.) At the Mount Holyoke meeting, Powers proposed the case when the CP-semigroups are spatial semigroups of unital endomorphisms where the units are isometric. In [Pow04, he generalized it to spatial CP-semigroups, but still with isometric units. He even calls these CP-semigroups spatial. But, we think that Arveson's (much) wider definition in Arv97. is the adequate one, and our discussion does already extend the Powers sum to that case.

In the preceding example we did not say a word about the product systems of the semigroups involved. In fact, the product system of $T^{n}$ consists of correspondences over $M_{n}(\mathcal{B})$, while the product system of the corresponding CPD-semigroup $\mathfrak{T}$ consists of correspondences over $\mathcal{B}$. For spatial product systems of correspondences over the same algebra $\mathcal{B}$, there is the product of spatial product systems. But, the algebras $M_{n}(\mathcal{B})$ may be nonisomorphic for different $n$. How are the product system of $T^{n}$ and of $\mathfrak{T}$ related, so that the product operation of the product system in the CPD-picture can be applied? Also the question whether Example 4.3 can be generalized to arbitrary index sets is interesting. We answer these and other questions in the more general setting of the following section.

5. The Powers sum of CP-semigroups on $\mathcal{B}^{a}(E)$. Observe that $M_{n}(\mathcal{B})=\mathcal{B}^{a}\left(\mathcal{B}^{n}\right)$. In this section we will replace $\mathcal{B}^{n}$ with a general full Hilbert $\mathcal{B}$-module (that is, $\overline{\operatorname{span}}\langle E, E\rangle$ $=\mathcal{B}$, respectively, $\overline{\operatorname{span}}^{s}\langle E, E\rangle=\mathcal{B}$ in the von Neumann case). But, then the terminology Schur CP-semigroup makes no longer sense. (This is something which makes sense only with respect to an ONB, like the canonical ONB of $\mathcal{B}^{n}$, or possibly a quasi ONB.) On the other hand, for the $C^{*}$-case in this setting it is indispensable that we require the CPsemigroups $T$ on $\mathcal{B}^{a}(E)$ to be strict, that is, each $T_{t}$ is $*$-strongly continuous on bounded subsets. (In the von Neumann case our standard hypothesis, normality, is sufficient.) The result that on $M_{n}(\mathcal{B})$ the strict topology coincides with the norm topology ( $\mathcal{B}$ is assumed unital!), is standard. This is why, in Example 4.3. we did not worry about strictness.

Before we study spatial CP-semigroups on $\mathcal{B}^{a}(E)$, we first repeat some results from Bhat, Liebscher, and Skeide BLS08a] about general strict CP-semigroups on $\mathcal{B}^{a}(E)$. We will also derive some new results on spatiality of such semigroups.

In BLS08a] we showed that the product system $F^{\odot}$ of a strict CP-semigroup $T$ on $\mathcal{B}^{a}(E)$ (consisting of correspondences over $\mathcal{B}^{a}(E)$ !) may be transformed into a product system $E^{\odot}$ consisting of correspondences over $\mathcal{B}$ in the following way: For each $F_{t}$ define the $\mathcal{B}$-correspondence $E_{t}:=E^{*} \odot F_{t} \odot E$, where $E^{*}$ is a correspondence from $\mathcal{B}$ to $\mathcal{B}^{a}(E)$ with inner product $\left\langle x^{*}, y^{*}\right\rangle:=x y^{*}$ (the rank-one operator that maps $z$ to $x\langle y, z\rangle$ ) and bimodule action $b x^{*} a:=\left(a^{*} x b^{*}\right)^{*}$. (Note that both tensor products are over $\mathcal{B}^{a}(E)$. Note, too, that $E^{*} \odot E=\mathcal{B}$ via $x^{*} \odot y=\langle x, y\rangle$ and $E \odot E^{*}=\mathcal{K}(E)$, the $C^{*}$-algebra 
of compact operators on $E$, via $x \odot y^{*}=x y^{*}$. Since all $T_{t}$ are strict, the left action of $\mathcal{K}(E)$ on $F_{t}$ is nondegenerate.) The $E_{t}$ form a product system $E^{\odot}$ via

$$
E_{s} \odot E_{t}=E^{*} \odot F_{s} \odot E \odot E^{*} \odot F_{t} \odot E=E^{*} \odot F_{s} \odot F_{t} \odot E=E^{*} \odot F_{s+t} \odot E=E_{s+t} .
$$

Note that when making disappear the part $E \odot E^{*}=\mathcal{K}(E)$ in the middle, we did use strictness of the left action on $F_{t}$. The right action of $\mathcal{K}(E)$ on $F_{s}$ will rarely be nondegenerate:

5.1. Remark. The transition $F^{\odot} \rightsquigarrow E^{\odot}$ is very close to an operation of Morita equivalence of product systems, as defined in Skeide Ske09b]. In fact, $E$ may be viewed as Morita equivalence from $\mathcal{K}(E)$ to $\mathcal{B}$. The inverse operation, conjugation of $E_{t}$ with $E$, gives $E \odot E_{t} \odot E^{*}=\overline{\operatorname{span}} \mathcal{K}(E) F_{t} \mathcal{K}(E)$. The left action of $\mathcal{K}(E)$ is nondegenerate by strictness. But, there is no reason why the inner product of $F_{t}$ should assume values only in $\mathcal{K}(E)$. In fact, if $T$ was a Markov semigroup, then each $F_{t}$ has a unit vector and $\mathcal{K}(E)$ is rarely unital.

When $T$ is an E-semigroup (that is, the maps $T_{t}$ are endomorphisms of $\mathcal{B}^{a}(E)$ ), or even an $E_{0}$-semigroup (that is, the endomorphisms are also unital), then the product system is the one associated in Skeide [Ske09b] with a strict E-semigroup. (See Skeide [Ske02 for the first construction for $E_{0}$-semigroup when $E$ has a unit vector, and Bhat and Lindsay [BL05] for an E-semigroup under the same hypothesis.) When $E=H$ is a Hilbert space, we recover Bhat's construction [Bha96] of the Arveson system of a normal $E_{0}$-semigroup on $\mathcal{B}(H)$. When $T$ is a normal CP-semigroup on $\mathcal{B}(G)$, we obtain a direct construction of its Arveson system. (In Bhat Bha96], it is constructed via the so-called minimal dilation of $T$ to an $E$-semigroup on $\mathcal{B}(H)$ as the Arveson system of that E-semigroup.)

The product system $E^{\odot}$ has no relation with $T$ as direct as the GNS-system $F^{\odot}$. (There is no unit for $E^{\odot}$ that would allow one to recover the CP-semigroup $T$. In fact, if $E=H$ is a Hilbert space, then it is known that $E^{\odot}$ can be unitless. In BLS08a, Theorem 3.4] we have shown that the product system of the minimal dilation is $E^{\odot}$.) But the following theorem shows that spatiality is preserved. For von Neumann modules this is a new result in the classification of product systems up to Morita equivalence; see Remark 5.4.

5.2. TheOREM. Let $E$ be a full Hilbert module over a unital $C^{*}$-algebra $\mathcal{B}$. Suppose $F^{\odot}$ is a product system of correspondences $F_{t}$ over $\mathcal{B}^{a}(E)$ with strict left actions. Define the product system $E^{\odot}$ as above by setting $E_{t}=E^{*} \odot F_{t} \odot E$.

If $F^{\odot}$ is spatial, then so is $E^{\odot}$. More precisely, if $\Omega^{\odot}$ is the central unital reference unit of $F^{\odot}$, then by $i_{t}:\langle x, y\rangle \mapsto x^{*} \odot \Omega_{t} \odot y \in E_{t}$ for each $t \in \mathbb{R}_{+}$, we define an injective morphism from the trivial product system $(\mathcal{B})_{t \in \mathbb{R}_{+}}$into $E^{\odot}$. In particular, the image of the central unital unit $(\mathbf{1})_{t \in \mathbb{R}_{+}}$is a central unital unit $\omega^{\odot}$ for $E^{\odot}$.

Proof. For each $t \in \mathbb{R}_{+}$, the map $i_{t}$ is an isometry. Indeed, $\left\langle x^{*} \odot \Omega_{t} \odot y, x^{\prime *} \odot \Omega_{t} \odot y^{\prime}\right\rangle=\left\langle\Omega_{t} \odot y, x x^{\prime *} \Omega_{t} \odot y^{\prime}\right\rangle=\left\langle\Omega_{t} \odot y, \Omega_{t} \odot x x^{\prime *} y^{\prime}\right\rangle=\langle y, x\rangle\left\langle x^{\prime}, y^{\prime}\right\rangle$.

Clearly, $i_{t}$ is bilinear. Since $\mathcal{B}$ is unital and $E$ is full, by [Ske09b, Lemma 3.2] there exist $n \in \mathbb{N}$ and $x_{1}, \ldots, x_{n} \in E$ such that $\sum_{i=1}^{n}\left\langle x_{i}, x_{i}\right\rangle=\mathbf{1}$. So, for $\omega_{t}:=i_{t}(\mathbf{1})=$ 
$\sum_{i=1}^{n} x_{i}^{*} \odot \Omega_{t} \odot x_{i}$ we find

$$
\begin{aligned}
\omega_{s} \odot \omega_{t}=\sum_{i, j=1}^{n} x_{i}^{*} \odot \Omega_{s} \odot x_{i} \odot x_{j}^{*} \odot \Omega_{t} \odot x_{j}=\sum_{i, j=1}^{n} x_{i}^{*} \odot \Omega_{s} \odot x_{i} x_{j}^{*} \Omega_{t} \odot x_{j} \\
=\sum_{i, j=1}^{n} x_{i}^{*} \odot \Omega_{s} \odot \Omega_{t} \odot x_{i} x_{j}^{*} x_{j}=\sum_{i=1}^{n} x_{i}^{*} \odot \Omega_{s+t} \odot x_{i}=\omega_{s+t},
\end{aligned}
$$

so that the $\omega_{t}$ form a unit $\omega^{\odot}$ for $E^{\odot}$. By bilinearity of $i_{t}$, the unit $\omega^{\odot}$ is unital and central. By the same reason, the $i_{t}$ form a morphism, that is, $i_{s} \odot i_{t}=i_{s+t}$.

5.3. Remark. A similar result is true for von Neumann modules. We just refer to Ske09b, Lemma 4.2], where the sum is no longer finite and, in general, only strongly convergent.

5.4. Remark. Note that the converse statement may fail. The simplest reason is that the correspondences $F_{t}$ of $F^{\odot}$ need not allow for unit vectors. More concretely, $F_{t}$ need not be strictly complete. (Otherwise, choose a bounded approximate unit for $\mathcal{K}(E)$ that consists of finite-rank operators $\sum_{i=1}^{n} x_{i} y_{i}^{*}$. Then the corresponding net of elements $\sum_{i=1}^{n} x_{i} \odot$ $\omega_{t} \odot y_{i}^{*}$ is strictly Cauchy in $F_{t}$. If the limits $\Omega_{t}$ exist, then they form a unital central unit for $F^{\odot}$.) For von Neumann modules also the converse statement is true: $E^{\odot}$ is spatial if and only if $F^{\odot}$ is spatial. (Von Neumann modules are not only strictly complete but even $*$-strongly.) In the sense of Morita equivalence of product systems of von Neumann correspondences Ske09b], one may rephrase this as follows: Morita equivalence of product systems of von Neumann correspondences preserves spatiality.

Now, since we know what is the product system $E^{\odot}$ of correspondences over $\mathcal{B}$ of a strict CP-semigroup $T$ on $\mathcal{B}^{a}(E)$ for some full Hilbert $\mathcal{B}$-module $E$, and since we know that spatiality of $T$ is reflected by spatiality of (some spatial extension of) $E^{\odot}$, we can ask whether there possibly is a Powers sum for spatial CP-semigroups such that the sum operation is reflected by the product operation of their spatial product systems of correspondences over $\mathcal{B}$. For $E_{0}$-semigroups we proved the affirmative answer in BLS08a]. For spatial CP-semigroups the result is new. The proof also simplifies the proof of [BLS08a].

We start with a simple consequence of Observation 2.4 .

5.5. Lemma. Let $E$ be a full Hilbert module over a unital $C^{*}$-algebra $\mathcal{B}$. Let $T$ be a spatial strict $C P$-semigroup on $\mathcal{B}^{a}(E)$ and choose a unit $S$ implemented by a semigroup $c$ in $\mathcal{B}^{a}(E)$. Denote by $F^{\odot}$ the spatial extension of the GNS-system associated with that unit as in the proof of Theorem 2.8 (considering $T$ a CPD-semigroup on a one-point set), so that $F^{\odot}$ is generated by the unit $\zeta^{\odot}$ that gives back $T_{t}=\left\langle\zeta_{t}, \bullet \zeta_{t}\right\rangle$ and by the central unital reference unit $\Omega^{\odot}$. Denote by $E^{\odot}$ and $\omega^{\odot}$ product system and central unit as in Theorem 5.2,

Then the product system $E^{\odot}$ is generated by elements of the form $\omega_{t}$ and $x^{*} \odot \zeta_{t} \odot y$ in the sense that

$$
E_{t}=\overline{\operatorname{span}}\left\{z_{n} \odot \ldots \odot z_{1}: n \in \mathbb{N}, t_{1}+\ldots+t_{n}=t, z_{i}=\omega_{t_{i}} \text { or } z_{i}=x_{i}^{*} \odot \zeta_{t_{i}} \odot y_{i}\right\} .
$$


We omit the obvious proof. Note, howewer, that

$$
\begin{aligned}
\left\langle x^{*} \odot \zeta_{t} \odot y, x^{\prime *} \odot \zeta_{t} \odot y^{\prime}\right\rangle & =\left\langle y, T_{t}\left(x x^{\prime *}\right) y^{\prime}\right\rangle, \\
\left\langle x^{*} \odot \zeta_{t} \odot y, x^{\prime *} \odot \Omega_{t} \odot y^{\prime}\right\rangle & =\left\langle y, c_{t}^{*} x\right\rangle\left\langle x^{\prime}, y\right\rangle,
\end{aligned}
$$

so that $\left\langle x^{*} \odot \zeta_{t} \odot y, \omega_{t}\right\rangle=\left\langle y, c_{t}^{*} x\right\rangle$. Note, too, that the pair $\left(E^{\odot}, \omega^{\odot}\right)$ is determined by these properties up to spatial isomorphism.

5.6. Theorem. For $i=1,2$, let $E^{i}$ be full Hilbert modules over a unital $C^{*}$-algebra $\mathcal{B}$, and let $T^{i}$ be a spatial strict CP-semigroup on $\mathcal{B}^{a}\left(E^{i}\right)$. Choose units $S^{i}$ for $T^{i}$ implemented by semigroups $c^{i}$ in $\mathcal{B}^{a}\left(E^{i}\right)$. Then, by setting

$$
\left(T^{1} \boxplus T^{2}\right)_{t}\left(\begin{array}{ll}
a_{11} & a_{12} \\
a_{21} & a_{22}
\end{array}\right):=\left(\begin{array}{ccc}
T_{t}^{1}\left(a_{11}\right) & c_{t}^{1^{*}} a_{12} c_{t}^{2} \\
c_{t}^{2 *} & a_{21} c_{t}^{1} & T_{t}^{2}\left(a_{22}\right)
\end{array}\right),
$$

we define a spatial CP-semigroup on $\mathcal{B}^{a}\left(E^{1} \oplus E^{2}\right)$ with a unit $S^{1} \boxplus S^{2}$ implemented by $c=c^{1} \oplus c^{2}$, the Powers sum $\left(T^{1} \boxplus T^{2}, S^{1} \boxplus S^{2}\right)$ of $\left(T^{1}, S^{1}\right)$ and $\left(T^{2}, S^{2}\right)$. Moreover, if $\left(E^{\odot}, \omega^{\odot}\right)$ denotes the spatial product system of correspondences over $\mathcal{B}$ associated with $\left(T^{1} \boxplus T^{2}, S^{1} \boxplus S^{2}\right)$, and if $\left(E^{i \odot}, \omega^{i \odot}\right)$ denote those associated with $\left(T^{i}, S^{i}\right)$, then $\left(E^{\odot}, \omega^{\odot}\right)=\left(E^{1} \odot E^{2}, \omega^{\odot}\right)$.

Proof. The proof that $T^{1} \boxplus T^{2}$ is a CP-semigroup, and that it is spatial with unit $c=$ $c^{1} \oplus c^{2}$ in $\mathcal{B}^{a}\left(E^{1} \oplus E^{2}\right)$, is similar to that of Theorem 3.1 .

To prove that $E^{\odot}$ is the product of $E^{1^{\odot}}$ and $E^{2} \odot$, we observe that by the lemma $E^{i \odot}$ is generated by expressions $\omega_{t}^{i}$ and $x^{i *} \odot \zeta^{i} \odot y^{i}$. Therefore the product $E^{1} \odot E^{2}$ is generated by expressions $\omega_{t}, x^{1^{*}} \odot \zeta^{1} \odot y^{1}$, and $x^{2^{*}} \odot \zeta^{2} \odot y^{2}$, where the only yet unspecified inner product is

$$
\left\langle x^{1^{*}} \odot \zeta^{1} \odot y^{1}, x^{2^{*}} \odot \zeta^{2} \odot y^{2}\right\rangle=\left\langle x^{1^{*}} \odot \zeta^{1} \odot y^{1}, \omega_{t}^{1}\right\rangle\left\langle\omega_{t}^{2}, x^{2^{*}} \odot \zeta^{2} \odot y^{2}\right\rangle=\left\langle y^{1}, c_{t}^{1^{*}} x^{1}\right\rangle\left\langle c_{t}^{2^{*}} x^{2}, y^{2}\right\rangle .
$$

On the other hand, $E^{\odot}$ is generated by expressions $\omega_{t}$ and $\left(\begin{array}{l}x^{1} \\ x^{2}\end{array}\right)^{*} \odot \zeta_{t} \odot\left(\begin{array}{l}y^{1} \\ y^{2}\end{array}\right)$. By calculating the norm, one easily verifies that $\left(\begin{array}{c}x^{1} \\ 0\end{array}\right)^{*} \odot \zeta_{t} \odot\left(\begin{array}{c}0 \\ y^{2}\end{array}\right)=\left(\begin{array}{c}0 \\ x^{2}\end{array}\right)^{*} \odot \zeta_{t} \odot\left(\begin{array}{c}y^{1} \\ 0\end{array}\right)=0$, while

$$
\begin{aligned}
& \left\langle\left(\begin{array}{c}
x^{1} \\
0
\end{array}\right)^{*} \odot \zeta_{t} \odot\left(\begin{array}{c}
y^{1} \\
0
\end{array}\right),\left(\begin{array}{c}
x^{1} \\
0
\end{array}\right)^{*} \odot \zeta_{t} \odot\left(\begin{array}{c}
y^{1} \\
0
\end{array}\right)\right\rangle=\left\langle x^{1^{*}} \odot \zeta_{t}^{1} \odot y^{1}, x^{1^{*}} \odot \zeta_{t}^{1} \odot y^{\prime 1}\right\rangle, \\
& \left\langle\left(\begin{array}{c}
0 \\
x^{2}
\end{array}\right)^{*} \odot \zeta_{t} \odot\left(\begin{array}{c}
0 \\
y^{2}
\end{array}\right),\left(\begin{array}{c}
0 \\
x^{\prime 2}
\end{array}\right)^{*} \odot \zeta_{t} \odot\left(\begin{array}{c}
0 \\
y^{\prime 2}
\end{array}\right)\right\rangle=\left\langle x^{2^{*}} \odot \zeta_{t}^{2} \odot y^{2}, x^{\prime 2 *} \odot \zeta_{t}^{2} \odot y^{\prime 2}\right\rangle, \\
& \left\langle\left(\begin{array}{c}
x^{1} \\
0
\end{array}\right)^{*} \odot \zeta_{t} \odot\left(\begin{array}{c}
y^{1} \\
0
\end{array}\right),\left(\begin{array}{c}
0 \\
x^{2}
\end{array}\right) * \zeta_{t} \odot\left(\begin{array}{c}
0 \\
y^{2}
\end{array}\right)\right\rangle=\left\langle x^{1^{*}} \odot \zeta_{t}^{1} \odot y^{1}, \omega_{t}^{1}\right\rangle\left\langle\omega_{t}^{2}, x^{2^{*}} \odot \zeta_{t}^{2} \odot y^{2}\right\rangle .
\end{aligned}
$$

So, $E^{\odot}$ is isomorphic to $E^{1} \odot E^{2}$, via

$$
\omega_{t} \mapsto \omega_{t},\left(\begin{array}{c}
x^{1} \\
0
\end{array}\right)^{*} \odot \zeta_{t} \odot\left(\begin{array}{c}
y^{1} \\
0
\end{array}\right) \mapsto x^{1^{*}} \odot \zeta_{t}^{1} \odot y^{1},\left(\begin{array}{c}
0 \\
x^{2}
\end{array}\right)^{*} \odot \zeta_{t} \odot\left(\begin{array}{c}
0 \\
y^{2}
\end{array}\right) \mapsto x^{2^{*}} \odot \zeta_{t}^{2} \odot y^{2} .
$$

5.7. REMARK. The algebras $\mathcal{B}^{a}\left(E^{1}\right)$ and $\mathcal{B}^{a}\left(E^{2}\right)$ have the property that they may be considered as subalgebras of the matrix algebra (see Skeide [Ske0] for details about matrix algebras) $\mathcal{B}^{a}\left(E^{1} \oplus E^{2}\right)=\left(\begin{array}{cc}\mathcal{B}^{a}\left(E^{1}\right) & \mathcal{B}^{a}\left(E^{2}, E^{1}\right) \\ \mathcal{B}^{a}\left(E^{1}, E^{2}\right) & \mathcal{B}^{a}\left(E^{2}\right)\end{array}\right)$. The interesting property is that both $\mathcal{B}^{a}\left(E^{1}\right)$ and $\mathcal{B}^{a}\left(E^{2}\right)$ are generated from products of the off-diagonal entries in the strict topology. (Note that this may fail if $E^{1}$ and $E^{2}$ are not both full, up to the point where $\mathcal{B}^{a}\left(E^{1}, E^{2}\right)=\{0\}$.) In the case of von Neumann modules that means that $\mathcal{B}^{a}\left(E^{1}\right)$ and $\mathcal{B}^{a}\left(E^{2}\right)$ are Morita equivalent as von Neumann algebras. (In fact, both are Morita equivalent to $\mathcal{B}$ as von Neumann algebras.) For $C^{*}$-modules one might say they are 
strictly Morita equivalent. (We do not know whether there exists a systematic study of Morita equivalence for multiplier algebras. This would be useful for our purposes, as the multiplier algebra of $\mathcal{K}(E)$ is $\mathcal{B}^{a}(E)$. We met already several times, in Ske09b, BLS08a], situations where we had to develop at least parts of such a theory.)

5.8. Remark. The case when $T^{i}$ are $E_{0}$-semigroups has been discussed in [BLS08a]. The proof here, restricted to that case, differs considerably from that in [BLS08a] and, actually, appears simpler. The case of $E_{0}$-semigroups acting on $\mathcal{B}(H) \mathrm{s}$ is the one proposed by Powers 2002 in Mount Holyoke; see also Example 4.3. The Arveson system of the Powers sum in that case has been identified as product in [Ske03a. The case of CPsemigroups acting on $\mathcal{B}(H)$ s has been discussed in Pow04 with a much less general notion of spatiality for CP-semigroups. Pow04 also does not identify the Arveson system of the sum as product of spatial Arveson systems. But, he proves that it need not be isomorphic to the tensor product. (The tensor product is available only for Arveson systems but not for modules.)

5.9. Remark. Of course, like in Remark 3.6, also here all statements remain true for families of spatial CP-semigroups and the spatial extensions of their GNS-systems.

6. CPD-semigroups versus Schur CP-semigroups. In Example 4.3 we pointed out that $\mathcal{B}(\mathcal{B})$-valued $\mathrm{CPD}$-semigroups on a finite set $S$ with cardinality $n$, say, are in one-to-one correspondence with Schur CP-semigroups on $M_{n}(\mathcal{B})$ and that this one-to-one correspondence behaves well with respect to the respective Powers sums. After Theorem 5.6 , we can say that this one-to-one correspondence also behaves well with respect to the products of the respective spatial extensions of the product systems of correspondences over $\mathcal{B}$. (They simply coincide.)

In this section we wish to see to what extent we can generalize that one-to-one correspondence to arbitrary sets $S$. The idea in Example 4.3 was to let the semigroups $\mathfrak{T}^{s, s^{\prime}}$ act on the matrix elements $a_{s, s^{\prime}}$ of a finite $\# S \times \# S$-matrix with entries in $\mathcal{B}$. We simply try now to do the same with $\# S \times \# S$-matrices for a set $S$ of arbitrary cardinality \#S.

Of course, the matrices should continue to form a $C^{*}$-algebra, so we cannot allow arbitrary matrices. A canonical candidate is the $C^{*}$-algebra $\mathcal{B}^{a}\left(\mathcal{B}^{S}\right)$ where $\mathcal{B}^{S}$ is the $\# S$-dimensional column space space of $\mathcal{B} . \mathcal{B}^{S}$ consists of all families $B=\left(b_{s}\right)_{s \in S}$ such that the net $\sum_{s \in S^{\prime}} b_{s}^{*} b_{s}$ converges over the finite subsets $S^{\prime}$ of $S$. The inner product is $\left\langle B, B^{\prime}\right\rangle:=\sum_{s \in S} b_{s}^{*} b_{s}^{\prime}$.

Let $e_{s}:=\left(\delta_{s s^{\prime}} \mathbf{1}\right)_{s^{\prime} \in S}$. The elements $e_{s}$ form an orthonormal basis of $\mathcal{B}^{S}$ in the obvious way: $\sum_{s \in S} e_{i} e_{i}^{*}=\mathrm{id}_{\mathcal{B}^{S}}$ strongly and, therefore, *-strongly in $\mathcal{B}^{a}\left(\mathcal{B}^{S}\right)$ over the finite subsets of $S$, and since the approximating net is bounded by $\mathbf{1}$, also strictly. It follows that an arbitrary element $A \in \mathcal{B}^{a}\left(\mathcal{B}^{S}\right)$ can be written as

$$
A=\sum_{s, s^{\prime} \in S} e_{s} a_{s, s^{\prime}} e_{s^{\prime}}^{*}
$$

where $a_{s, s^{\prime}}:=\left\langle e_{s}, A e_{s^{\prime}}\right\rangle \in \mathcal{B}$. (We resist the temptation to denote $\mathcal{B}^{a}\left(\mathcal{B}^{S}\right)$ as $M_{S}(\mathcal{B})$, because the latter, usually, rather refers to $\mathcal{K}\left(\mathcal{B}^{S}\right)$.) 
A Schur CP-map on $\mathcal{B}^{a}\left(\mathcal{B}^{S}\right)$ is a CP-map $T$ on $\mathcal{B}^{a}\left(\mathcal{B}^{S}\right)$ such that

$$
T\left(e_{s} e_{s}^{*} A e_{s^{\prime}} e_{s^{\prime}}^{*}\right)=e_{s} e_{s}^{*} T(A) e_{s^{\prime}} e_{s^{\prime}}^{*}
$$

for all $A \in \mathcal{B}^{a}\left(\mathcal{B}^{S}\right)$ and all $s, s^{\prime} \in S$. Without its simple proof, we state the following:

6.1. Proposition. A Schur CP-map necessarily leaves $\mathcal{K}\left(\mathcal{B}^{S}\right)$ invariant and is strict.

Obviously, if $T$ is a Schur CP-map, then $\mathfrak{K}^{s, s^{\prime}}:=\left\langle e_{s}, T\left(e_{s} \bullet e_{s^{\prime}}^{*}\right) e_{s^{\prime}}\right\rangle$ defines a $\mathcal{B}(\mathcal{B})$-valued CPD-kernel $\mathfrak{K}$ on $S$. Moreover, $T$ can be recovered from $\mathfrak{K}$ as

$$
T\left(e_{s} b e_{s^{\prime}}^{*}\right)=e_{s} \mathfrak{K}^{s, s^{\prime}}(b) e_{s^{\prime}}^{*} .
$$

However, not all CPD-kernels give rise to Schur CP-map in that way. A CPD-kernel is bounded if there is a constant $M$ such that $\left\|\mathfrak{K}^{s, s^{\prime}}\right\| \leq M$ for all $s, s^{\prime} \in S$.

6.2. Proposition. Let $\mathfrak{K}$ be a $\mathcal{B}(\mathcal{B})$-valued $C P D$-kernel $\mathfrak{K}$ on $S$. Then $\mathfrak{K}$ gives rise to a (unique) Schur CP-map on $\mathcal{B}^{a}\left(\mathcal{B}^{S}\right)$ fulfilling (6.1) if and only if $\mathfrak{K}$ is bounded.

Proof. Clearly, a kernel $\mathfrak{K}$ fulfilling (6.1) for some CP-map $T$, is bounded by $M=\|T\|$. So, for the other direction let us suppose that $\mathfrak{K}$ is bounded (by $M$, say). Instead of showing directly that under this condition the map defined by (6.1) on finite matrices (that is, operators $A \in \mathcal{B}^{a}\left(\mathcal{B}^{S}\right)$ with only finitely many matrix entries $a_{s, s^{\prime}}$ different from 0) extends suitably to a CP-map $T$, we shall construct a candidate for the GNSconstruction of $T$.

Let $\left(E,\left(\xi^{s}\right)_{s \in S}\right)$ denote the Kolmogorov decomposition for $\mathfrak{K}$. Define $F:=\mathcal{B}^{S} \odot E \odot \mathcal{B}_{S}$, where $\mathcal{B}_{S}:=\mathcal{B}^{S^{*}}$, the $\# S$-dimensional row space of $\mathcal{B}$. Recall that an element $y \in F$ may be interpreted as a map $B \mapsto y \odot B$ from $\mathcal{B}^{S}$ to $F \odot \mathcal{B}^{S}=\mathcal{B}^{S} \odot E$. We claim that the sum $\sum_{s \in S} e_{s} \odot \xi^{s} \odot e_{s}^{*}$ converges $*$-strongly in $\mathcal{B}^{a}\left(\mathcal{B}^{S}, \mathcal{B}^{S} \odot E\right)$ to an operator $Z$. Once we have convergence, it is clear that the CP-map $T(A):=Z^{*}\left(A \odot \operatorname{id}_{E}\right) Z$ fulfills 6.1).

Let $B=\left(b_{s}\right)_{s \in S} \in \mathcal{B}^{S}$. Then for every finite subset $S^{\prime} \subset S$ we have

$$
\left|\sum_{s \in S^{\prime}}\left(e_{s} \odot \xi^{s} \odot e_{s}^{*}\right) B\right|^{2}=\left|\sum_{s \in S^{\prime}} e_{s} \odot \xi^{s} b_{s}\right|^{2}=\sum_{s \in S^{\prime}} b_{s}^{*}\left\langle\xi^{s}, \xi^{s}\right\rangle b_{s} \leq M \sum_{s \in S^{\prime}} b_{s}^{*} b_{s} .
$$

From this, two things follow. Firstly, $\sum_{s \in S^{\prime}}\left(e_{s} \odot \xi^{s} \odot e_{s}^{*}\right) B$ is a Cauchy net in $\mathcal{B}^{S} \odot E$. Secondly, the net $\sum_{s \in S^{\prime}} e_{s} \odot \xi^{s} \odot e_{s}^{*}$ is bounded by $\sqrt{M}$. From boundedness it follows that strong convergence of the adjoint net may be checked on the total subset $e_{s} \odot x$ of $\mathcal{B}^{S} \odot E$. But this is clear because $\sum_{s \in S^{\prime}}\left(e_{s} \odot \xi^{s} \odot e_{s}^{*}\right)^{*}\left(e_{s^{\prime}} \odot x\right)=e_{s^{\prime}}\left\langle\xi^{s^{\prime}}, x\right\rangle$ if $s^{\prime} \in S^{\prime}$ and 0 otherwise.

6.3. Corollary. Fix a set $S$ and a unital $C^{*}$-algebra $\mathcal{B}$. Then the formula (6.1), when applied to all members of a semigroup, establishes a one-to-one correspondence between pointwise bounded $\mathcal{B}(\mathcal{B})$-valued $C P D$-semigroups $\mathfrak{T}$ on $S$ (that is, each $\mathfrak{T}_{t}$ is bounded) and Schur CP-semigroups $T$ on $\mathcal{B}^{S}$. Moreover, $\mathfrak{T}$ and $T$ have the same product systems of correspondences over $\mathcal{B}$.

Proof. We omit the proof of the only open statement, that about the product systems. (It follows from the observation that the unit $\zeta^{\odot}$ of the GNS-system of $T$ is a diagonal matrix with the unit $\xi^{\odot} \odot$ of the GNS-system of $\mathfrak{T}$ as $s, s$-entry; see also [BBLS04, Appendix B].) 
6.4. TheOREM. $\mathfrak{T}$ is spatial if and only if $T$ is spatial. In that case, the one-to-one correspondence also respects units and the spatial extensions of the GNS-systems based on them. Therefore, it must also respect Powers sums (and obviously products of the product systems, because the product systems coincide, anyway).

We omit the obvious proof also here.

6.5. Remark. The formulation is for $C^{*}$-algebras and modules. A similar correspondence has been proved for von Neumann algebras and modules in [BBLS04, Appendix B], however, only for uniformly continuous semigroups, and without paying attention to the one-to-one aspect and the related notion of Schur CP-semigroup. (Recall that uniform continuity is automatic only for spatial semigroups. The statements that are valid also in the nonspatial case, do not require any continuity in time.) Anyway, all statements remain true also in the von Neumann case, some of them simpler, because no spatial extension is needed.

6.6. REMARK. Note that for pointwise bounded CPD-semigroups, the results in Section 3 may be obtained from those in Section 5 via the one-to-one correspondence. (The only exception is the spatial extension of the GNS-system of a spatial CP-semigroup. But this can easily be added to Section 5, to make it independent of Section 3.) The non-pointwise bounded case can also be reduced to the pointwise bounded case, by rescaling the CPDsemigroup with scalar semigroups. But this discussion is somewhat cumbersome and not at all instructive. We prefer to leave Section 3 as a separate one, which in its general form is not included in Section 5 .

Acknowledgments. This work is supported by research funds of of the Dipartimento S.E.G.e S. and Italian MUR under PRIN 2007.

\section{References}

[Arv89] W. Arveson, Continuous analogues of Fock space III: Singular states, J. Operator Theory 22 (1989), 165-205.

[Arv97] W. Arveson, The index of a quantum dynamical semigroup, J. Funct. Anal. 146 (1997), 557-588.

[BBLS04] S. D. Barreto, B. V. R. Bhat, V. Liebscher, and M. Skeide, Type I product systems of Hilbert modules, J. Funct. Anal. 212 (2004), 121-181.

[Bha96] B. V. R. Bhat, An index theory for quantum dynamical semigroups, Trans. Amer. Math. Soc. 348 (1996), 561-583.

[BL05] B. V. R. Bhat and J. M. Lindsay, Regular quantum stochastic cocycles have exponential product systems, in: Quantum Probability and Infinite Dimensional Analysis From Foundations to Applications, M. Schürmann and U. Franz (eds.), Quantum Probability and White Noise Analysis XVIII, World Scientific, 2005, 126-140.

[BLS08a] B. V. R. Bhat, V. Liebscher, and M. Skeide, A problem of Powers and the product of spatial product systems, in: Quantum Probability and Infinite Dimensional Analysis - Proceedings of the 28th Conference, J. C. Garcia et al. (eds.), Quantum Probability and White Noise Analysis XXIII, World Scientific, 2008, 93-106. 
[BLS08b] B. V. R. Bhat, V. Liebscher, and M. Skeide, Subsystems of Fock need not be Fock: Spatial CP-semigroups, arXiv: 0804.2169v1, 2008.

[BS00] B. V. R. Bhat and M. Skeide, Tensor product systems of Hilbert modules and dilations of completely positive semigroups, Infin. Dimens. Anal. Quantum Probab. Relat. Top. 3 (2000), 519-575.

[Lie03] V. Liebscher, Random sets and invariants for (type II) continuous tensor product systems of Hilbert spaces, Mem. Amer. Math. Soc. 199 (2009), no. 930.

[Pas73] W. L. Paschke, Inner product modules over $B^{*}$-algebras, Trans. Amer. Math. Soc. 182 (1973), 443-468.

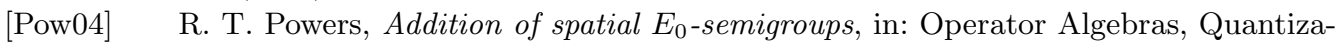
tion, and Noncommutative Geometry, Contemporary Mathematics 365, American Mathematical Society, 2004, 281-298.

[PS72] K. R. Parthasarathy and K. Schmidt, Positive Definite Kernels, Continuous Tensor Products, and Central Limit Theorems of Probability Theory, Lect. Notes Math. 272, Springer, 1972.

[Ske00] M. Skeide, Generalized matrix $C^{*}$-algebras and representations of Hilbert modules, Mathematical Proceedings of the Royal Irish Academy 100A (2000), 11-38.

[Ske02] M. Skeide, Dilations, product systems and weak dilations, Math. Notes 71 (2002), 914-923.

[Ske03a] M. Skeide, Commutants of von Neumann modules, representations of $\mathcal{B}^{a}(E)$ and other topics related to product systems of Hilbert modules, in: Advances in Quantum Dynamics, G. L. Price et al. (eds.), Contemporary Mathematics 335, American Mathematical Society, 2003, 253-262.

[Ske03b] M. Skeide, Dilation theory and continuous tensor product systems of Hilbert modules, in: Quantum Probability and Infinite Dimensional Analysis, W. Freudenberg (ed.), Quantum Probability and White Noise Analysis XV, World Scientific, 2003, 215242 .

[Ske06] M. Skeide, The index of (white) noises and their product systems, Infin. Dimens. Anal. Quantum Probab. Relat. Top. 9 (2006), 617-655.

[Ske08] M. Skeide, Isometric dilations of representations of product systems via commutants, Int. J. Math. 19 (2008), 521-539.

[Ske09a] M. Skeide, Classification of $E_{0}$-semigroups by product systems, arXiv: 0901.1798v1, 2009.

[Ske09b] M. Skeide, Unit vectors, Morita equivalence and endomorphisms, Publ. Res. Inst. Math. Sci. 45 (2009), 475-518. 
